\title{
A Mindfulness-Based Stress Reduction Program for Anesthesiology Residents Evaluated with Biophysical Markers of Stress and Validated Burnout Assessments
}

\section{Christopher Cowart}

Mount Sinai Health System

Lauren Lisann-Goldman ( $\sim$ lauren.r.lisann@gmail.com )

Mount Sinai Health System

Hung-Mo Lin

Mount Sinai Health System

Jerrold Meyer

University of Massachusetts Amherst

Barbara Orlando

Mount Sinai Health System

Bryan Mahoney

Mount Sinai Health System

\section{Research Article}

Keywords: mindfulness, burnout, wellness, stress

Posted Date: May 24th, 2021

DOI: https://doi.org/10.21203/rs.3.rs-531998/v1

License: (1) (1) This work is licensed under a Creative Commons Attribution 4.0 International License. Read Full License 


\section{Abstract}

Background: Burnout has negative psychological and physical consequences for physicians in training. Mindfulness-based stress reduction (MBSR) has a promising track record for improving well-being. This study aims to demonstrate biophysical and psychological benefit from the incorporation of MBSR into the curriculum of an urban anesthesiology residency program.

Methods: This prospective cohort study compared the effect of a voluntary 18-month (January 2018 June 2019) biweekly MBSR course to an active control (availability of virtual cognitive behavioral therapy (CBT) curriculum and app-based mindfulness tools). Biometric data (e.g., sleep quantity and quality, physical activity and heart rate variability) from wearable devices; hair cortisol levels, and Maslach Burnout Inventory scores were compared between and within cohorts.

Results: Data collection was discontinued at the end of the first year of the study due to poor utilization of the in person MBSR and virtual/ app-based trainings. Of 76 eligible anesthesiology trainees, 38 participated (50\% of total eligible). Depersonalization scores were significantly lower in the MSBR group. Emotional exhaustion and depersonalization scores were significantly higher for clinical anesthesia (CA) CA-2 (post-graduate year (PGY)-3) than CA-1 (PGY-2) residents. There were no significant differences between cohorts for biophysical outcomes.

Conclusions: The implementation of an in-person MBSR curriculum for anesthesiology residents in an urban setting suffered from low utilization. Depersonalization scores were significantly lower in the MBSR compared to the active control group. Perioperative training programs may find more utility in wellness initiatives that are not reliant on inflexibly scheduled courses that require additional time commitment on the part of trainees.

\section{Background}

Burnout, "mental and physical exhaustion related to work or caregiving," ${ }^{1}$ affects $27-75 \%$ of physicians in training. ${ }^{2}$ This manifests as worsening mental health, ${ }^{3}$ cardiovascular disease, ${ }^{4}$ poor sleep hygiene, ${ }^{5}$ and malnutrition, and has a prevalence in anesthesiology between $40-50 \%,{ }^{6,7}$ Burnout may negatively affect patient care, and has particular importance in anesthesiology given the high rate of substance abuse and suicide amongst anesthesiologists. ${ }^{8-10}$

The American Council of Graduate Medical Education (ACGME) recently mandated that accredited programs address resident well-being, guided by the rationale that psychological and physical well-being are requisite to the development of resilient physicians. ${ }^{11}$ Anesthesiology training involves many potential challenges: production pressure, isolation from colleagues, and a perceived lack of respect from surgeons. ${ }^{12}$

Multiple targets exist for reducing burnout, ${ }^{13}$ with proven interventions including organizational initiatives focused on duty hours and workflow, along with individual-based strategies including mindfulness-based 
stress reduction (MBSR) or sessions on stress management. ${ }^{14}$ Despite a growing call for structural or organizational change, ${ }^{15}$ many programs continue to face economic or institutional barriers and are compelled to rely instead upon individual-targeted approaches.

The benefits of mindfulness-based curricula have demonstrated benefit in burnout prevention, though results are mixed in the setting of implementation in anesthesiology residency. ${ }^{14,16,17}$ Attitudes amongst trainees in perioperative sub-specialties towards these interventions may temper their effectiveness. ${ }^{18}$ No studies currently exist investigating the feasibility of in-person MBSR for addressing burnout among anesthesiology trainees. This study aims to evaluate the effects of MBSR on resident well-being and burnout, as measured by hair cortisol levels, Maslach Burnout Inventory (MBI) scores and sleep status level (SSL).

\section{Methods}

\section{Study Design}

Institutional Review Board (IRB) approval was obtained prior to this prospective cohort study. All methods were performed in accordance with the relevant guidelines and regulations. Participants included trainees in two urban anesthesiology training programs within a single health care system. The intervention group consisted of residents from one training program for whom in-person MBSR training was made available; the "active" control consisted of residents from a second training program within the same health care system for whom an online cognitive behavioral therapy (CBT) course and app-based mindfulness resources were made available. This design is based on the body of research on mindfulness and MBSR employing both wait-list and "active" controls. 19,20

Inclusion criteria included CA-1 or CA-2 training level (PGY-2 and 3), resulting in 76 total eligible anesthesiology residents. Exclusion criteria included PGY-1 and PGY-4 training level given the inability of interns to consistently attend and the inability of those at the PGY-4 training level to complete the twoyear study protocol.

After obtaining consent, study participants were to be followed over a 24-month period including a 3month pre-intervention phase, 18-month intervention phase and 3-month post-intervention phase. Primary outcome variables included Maslach Burnout Inventory - Human Services Survey (MBI-HSS) scores, hair cortisol level and sleep status level (SSL) collected through Fitbit Alta HR ${ }^{\mathrm{TM}}$ (Fitbit Inc., San Francisco, CA) wearable devices.

Intervention: The in-person training cohort was provided a MBSR course every other week over 18 months (January 2018 - June 2019) during morning lecture time (6:30 to 7:00 AM) at a single training program. The MBSR curriculum was based on the original course developed by Jon Kabat-Zinn at the University of Massachusetts. ${ }^{21}$ The curriculum provided to our participants was modified to consist of 36 half-hour sessions over 18 months (deviating from weekly 2.5 hour sessions over 8 weeks originally described for 
MSBR), due to scheduling restrictions imposed by operating room staffing requirements. MSBR sessions consisted of instruction in sitting meditation, mindful movement, body scan, and walking meditation. Generally, the aim of the course and these exercises is to learn about and recognize stress to modify its effects by applying mindfulness techniques to daily life. Session attendance was optional and available to all anesthesiology house staff in the program to which it was provided. The instructor was certified to provide instruction in MSBR and was hired through the Graduate Medical Education office to all training programs in our healthcare system.

The control group received access to moodgym ${ }^{\circledR}$ (ehubHealth Pty Ltd., Goulburn, AU), an app-based CBT training product, and InsightTimer@ (Insight Network Inc., San Francisco, CA) an app-based source of meditation timers and mindfulness meditation tracks. Use of these products was voluntary and not recorded to preserve subjects' privacy.

\section{Data Collection}

Maslach Burnout Inventory - Human Services Survey (MBI-HSS) and hair cortisol levels were obtained on day 1 of the pre-intervention phase, at which time SSL data collection with Fitbit Alta $\mathrm{HR}^{\mathrm{TM}}$ devices was also initiated. The proposed data collection schedule is presented in Table 1.

The MBI-HSS, a validated 22-question survey, is the most commonly used tool for measuring burnout in clinicians. ${ }^{22}$ It consists of 3 scales that assess three domains of burnout: emotional exhaustion, depersonalization, and personal accomplishment. ${ }^{23}$

Hair cortisol level testing is a surrogate marker of stress, given that stress is associated with increased activity of the hypothalamic-pituitary-adrenal axis. It is an easily accessible means of cortisol testing when examining human cortisol levels over a period of several months, although some studies suggest that in order to detect cortisol levels in a given hair sample the stress must be ongoing. ${ }^{24}$ Hair was cut as close to the scalp as possible with clean scissors in an amount of hair roughly the width of a pencil from the posterior vertex of the head. Samples $3 \mathrm{~cm}$ in length representing cortisol deposition over roughly the 3-month period prior to collection were obtained by using a ruler to measure the desired length from the cut end and cut again to yield the correct sample. Samples were wrapped in aluminum foil, labeled with a permanent marker with each participant's unique identification number, stored at room temperature, then shipped overnight to the University of Massachusetts, to the lab that developed this method for cortisol analysis. $^{25}$

Sleep State Level was measured through the Fitbit Alta $\mathrm{HR}^{\mathrm{TM}}$ electronic fitness tracker, which each participant was asked to wear 24 hours a day, except when charging. This device is known to be safe in the operating room without interfering with equipment or monitors. Each participant was asked to download Detalytics@ (Detalytics Pte. Ltd, Singapore), an app that would allow data from the Fitbit to be uploaded and analyzed. The Detalytics@ proprietary sleep analytics suite analyzes the level and stability of sleep during a single sleep cycle and across multiple episodes of sleep. The three data endpoints comprise the Sleep Status Level: sleep quantity (duration of time the participant is asleep), sleep quality 
(time required to fall asleep and the number of awakenings experienced in a single sleep cycle), and sleep consistency (overall variability within multiple sleep cycle measurements).

\section{Data Analysis}

The primary objective of the analysis was to evaluate the effect of MBSR on Maslach results and hair cortisol at three time points or campaigns (baseline, 180 days, and 360 days), and to determine whether there existed a change over time. The secondary objective was to investigate the effect of residency year overall and its trend. A mixed model was used with fixed effects including intervention group, campaign time (baseline, 180 and 360 days), clinical anesthesia year (CA-1 and CA-2), interaction between intervention group and time, and interaction between clinical anesthesia year and time. Random effects included the study subjects. The mixed model accounted for the within-subject correlation of the repeated assessments during the campaign periods.

Data are presented as median [interquartile range] and $\mathrm{N}(\%)$. For comparisons of Fitbit Alta ${ }^{\mathrm{TM}} \mathrm{HR}$ and sleep data between the intervention and control groups, the Wilcoxon-Mann Whitney test was used for continuous data and Fisher's exact test was used for categorical data where appropriate. In general, a 2sided $p$-value of $<0.05$ signifies statistical differences between the two groups. Although many data points were collected by the Fitbit Alta ${ }^{\text {TM }}$ devices, the study sample size was limited. Therefore, we chose to use 0.01 as the significance criterion instead of correcting the p-values for the multiple comparisons. The statistical analysis was performed using SAS Software (Version 9.4, SAS Institute, Cary, NC, USA).

\section{Results}

Of the total 76 eligible residents, 38 chose to participate, including both the intervention and control groups ( $50 \%$ of eligible participants). The control group consisted of 10 CA- 1 and 8 CA- 2 residents; the intervention group consisted of $10 \mathrm{CA}-1$ and $10 \mathrm{CA}-2$ residents $(P=0.478)$. Data collection was discontinued after one year rather than two due to low utilization of the MBSR in-person training and appbased resources.

\section{MBI-HSS:}

The Maslach Burnout Inventory for medical personnel consists of three domains, Emotional Exhaustion, Depersonalization, and Personal Accomplishment. The results from each domain are scored on a 7-level scale from $0-6$, with higher scores denoting worse burnout.

Emotional Exhaustion (Fig. 1, Table 2):

There was no significant difference between intervention and control groups on Maslach emotional exhaustion total score at all-time points. However, significant interaction existed between time and clinical anesthesia year $(p=0.048)$. The emotional exhaustion total score was higher for CA-2s at 180 days (Diff (SE): 7.81 (3.73); $p=0.047)$ ) and 360 days (8.08 (4.75); $p=0.101)$, when compared to CA-1s. This 
difference was not significant at baseline prior to the start of the mindfulness intervention (-0.1902 (2.98); $\mathrm{p}=0.950)$.

Depersonalization (Fig. 2, Table 2):

Depersonalization total score was consistently lower for the intervention group by -3.90 on average (1.82); $p=0.040$ ). As with emotional exhaustion total score, the interaction between time and clinical anesthesia year was significant $(p=0.002)$. Depersonalization total score was higher for $C A-2 s$ by $5.40(2.25)(P=$ $0.024)$ at 180 days. This difference was not significant at baseline prior to the start of the mindfulness intervention (-2.01 (1.90); $p=0.301)$ or at 360 days (0.84 (2.74); $p=0.762)$. At 180 days, depersonalization score declined for CA-1's $(-0.77(0.26) ; P=0.007)$ but increased for CA-2s $(0.72(0.25) ; P=0.009)$, compared to baseline. This trend was not observed at 360 days.

Personal Accomplishment (Fig. 3, Table 2):

A significant difference in personal accomplishment total scores existed between CA-1s and CA-2s at day 180 and 360 (respectively, -5.85 (2.23); $P=0.015$ at day 180 and -6.35 (2.84); $P=0.034$ at day 360; interaction between $C A$ year and time $P=0.033)$. There was also a significant difference in personal accomplishment total scores over time between baseline and day 180 in CA-2s (-3.09 (1.43); $P=0.040)$. No other meaningful comparisons over time or between groups for personal accomplishment scores were significant.

Fitbit Alta HR ${ }^{\mathrm{m}}$ : Data from the Fitbit Alta $\mathrm{HR}^{\mathrm{TM}}$ showed no significant difference between the intervention and control groups with the exception of minutes spent in "Zone 2" heart rate (intervention: median 285 minutes [IQR: 265-370] and control: 222 minutes [IQR: 183-498], Wilcoxon rank sum test $p=0.032$ ), and time spent performing vigorous exercise (Intervention: 4.01 minutes [3.52-5.76] vs. control 16.46 [9.9831.23], $p=0.032$.

\section{Hair Cortisol}

There was no significant difference in hair cortisol levels when between the control and intervention groups at any time throughout the study.

\section{Discussion}

Our experience conducting a two-year study to assess the effectiveness of in-person MBSR training for anesthesiology residents revealed many difficulties in implementation with few significant benefits (as measured by MBI-HSS scores and biometric data). The in-person and app-based resources were made available to our two cohorts, though utilization was not mandated or tracked to protect subjects' anonymity, given that all of the interventions were mental health-related. Furthermore, the nature of residency training makes consistent attendance difficult when accounting for subspecialty and off-site rotations, call schedules, night shifts, vacations and conference attendance. More importantly, attendance was stymied by participants' lack of buy-in when introduced to MBSR. While data collection of MBI-HSS 
and hair cortisol levels was discontinued after one year of the study, the MBSR in-person training as well as app-based training continued to be offered through the duration of the original study design.

The significant difference in depersonalization scores between the intervention and control groups likely represents selection bias inherent in our study design, given that this difference existed at the outset and persisted over time. Findings related to clinical training level likely reflect realities of anesthesiology training unrelated to our study intervention. CA-1 year represents the steepest learning curve of residency spent primarily in the practice of general anesthesiology. Most CA-1 residents achieve a relative level of comfort by the second half of this year with an accompanying sense of capability. The CA-2 year of training, however, is commonly a year of new subspecialty rotations providing challenges following the relative confidence achieved by the end of the first year of training. Our findings of decreased emotional exhaustion and increased personal accomplishment over time in CA-1 residents with the opposite pattern in CA-2 residents may reflect this anecdotally reported difference in experience between these two years of training (Figs. 1 and 3). The existence of significant trends in these two domains highlights particular time points that might be targeted in future interventions (whether at the individual, departmental, or institutional level).

Our study was motivated by a perceived need to address burnout, now tracked through ACGME annual surveys and internal evaluations by our health care system Graduate Medical Education office. We quickly found that attempting to reduce burnout with individual-targeted offerings without enacting structural change to address the drivers of burnout will not reduce burnout. In reality, the solution is likely to require interventions at all levels cited as potential sources for drivers of burnout (i.e. individual interventions, departmental and institutional restructuring, and even profession-wide change). While we did not conduct surveys to assess resident reception, many comments were freely shared. Not only was there a general lack of interest in MBSR among our house staff, but residents resented that our wellness intervention represented an additional time commitment, and attendance was accordingly poor.

Our study had many additional limitations. The inability to randomize trainees was necessitated by the logistics of delivering these interventions, resulting in a cohort design, leading to selection bias. Further, not tracking attendance or app usage limits our ability to attribute significant and non-significant results to the intervention. Inconsistent wearable device utilization weakens the validity of SSL, HR "zone" and physical exercise findings. Finally, many of the challenges we faced in providing MBSR in-person training may be unique to programs in an urban setting.

\section{Conclusions}

Setting aside time that would otherwise be spent in clinical duties rather than during didactic or free time may improve resident reception of time-intensive interventions. Further, a combination of individual and organizational changes that address a variety of the drivers of burnout may be more effective than interventions purely targeted at individuals. The experience of this group will be informative for other perioperative programs seeking to implement initiatives to combat resident burnout. 


\section{Abbreviations}

Mindfulness-based stress reduction (MBSR); cognitive behavioral therapy (CBT); clinical anesthesia (CA); post-graduate year (PGY); American Council of Graduate Medical Education (ACGME); Maslach Burnout Inventory (MBI); sleep status level (SSL); Maslach Burnout Inventory - Human Services Survey (MBI-HSS)

\section{Declarations}

Ethics approval and consent to participate: Mount Sinai Health System Institutional Review Board (IRB) approval was obtained prior to this prospective cohort study. Informed consent was obtained from all subjects.

Consent for publication: Not applicable - no individual person's data is included in any form.

Availability of data and materials: The datasets used and analyzed are available from the corresponding author.

Competing interests: The authors declare that they have no competing interests.

Funding: Funding was provided by the Icahn School of Medicine Eliasberg Scholarship. The funding body was not involved in the study design, data collection, analysis or interpretation, or in writing of the manuscript.

Authors' contributions: Christopher Cowart, M.D. - data collection and analysis, manuscript preparation. Lauren Lisann-Goldman, M.D. - data collection and analysis, manuscript preparation. Hung-Mo Lin, Sc.D. data analysis and manuscript preparation. Jerrold S. Meyer, Ph.D. - data collection. Barbara Orlando, M.D. - data collection and analysis, manuscript preparation. Bryan Mahoney, M.D. - study Design, manuscript preparation.

Acknowledgements: We would like to thank Mickie Brown for MBSR teaching throughout the study. Authors' information: Not applicable.

\section{References}

1. Freudenberger HJ. The staff burnout. J Soc Issues. Published online 1974.

2. IsHak WW, Lederer S, Mandili C, et al. Burnout During Residency Training: A Literature Review. J Grad Med Educ. Published online 2009. doi:10.4300/jgme-d-09-00054.1

3. Grossi G, Perski A, Osika W, Savic I. Stress-related exhaustion disorder - clinical manifestation of burnout? A review of assessment methods, sleep impairments, cognitive disturbances, and neurobiological and physiological changes in clinical burnout. Scand J Psychol. Published online 2015. doi:10.1111/sjop.12251 
4. Melamed S, Shirom A, Toker S, Berliner S, Shapira I. Burnout and risk of cardiovascular disease: Evidence, possible causal paths, and promising research directions. Psychol Bull. Published online 2006. doi:10.1037/0033-2909.132.3.327

5. Melo MCA, Das Chagas Medeiros F, De Bruin VMS, Santana JAP, Lima AB, De Francesco Daher E. Sleep quality among psychiatry residents. Can J Psychiatry. Published online 2016. doi:10.1177/0706743715620410

6. Hamidi MS, Boggild MK, Cheung AM. Running on empty: A review of nutrition and physicians' wellbeing. Postgrad Med J. 2016;92(1090):478-481. doi:10.1136/postgradmedj-2016-134131

7. Hyman SA, Michaels DR, Berry JM, Schildcrout JS, Mercaldo ND, Weinger MB. Risk of burnout in perioperative clinicians: A survey study and literature review. Anesthesiology. Published online 2011. doi:10.1097/ALN.0b013e318201ce9a

8. De Oliveira GS, Chang R, Fitzgerald PC, et al. The prevalence of burnout and depression and their association with adherence to safety and practice standards: A survey of united states anesthesiology trainees. Anesth Analg. Published online 2013. doi:10.1213/ANE.0b013e3182917da9

9. Bryson EO, Silverstein JH. Addiction and substance abuse in anesthesiology. Anesthesiology. Published online 2008. doi:10.1097/ALN.0b013e3181895bc1

10. Alexander BH, Checkoway H, Nagahama SI, Domino KB. Cause-specific mortality risks of anethesiologists. Anesthesiology. Published online 2000. doi:10.1097/00000542-200010000-00008

11. Accreditation Council for Graduate Medical Education. ACGME Common Program Requirements (Residency):; 2020.

12. Kuhn CM, Flanagan EM. Self-care as a professional imperative: physician burnout, depression, and suicide. Can J Anesth. Published online 2017. doi:10.1007/s12630-016-0781-0

13. Shanafelt TD, Noseworthy JH. Executive Leadership and Physician Well-being: Nine Organizational Strategies to Promote Engagement and Reduce Burnout. Mayo Clin Proc. Published online 2017. doi:10.1016/j.mayocp.2016.10.004

14. West CP, Dyrbye LN, Erwin PJ, Shanafelt TD. Interventions to prevent and reduce physician burnout: a systematic review and meta-analysis. Lancet. Published online 2016. doi:10.1016/S01406736(16)31279-X

15. Wolpaw JT. It Is Time to Prioritize Education and Well-Being Over Workforce Needs in Residency Training. Acad Med. Published online 2019. doi:10.1097/ACM.0000000000002949

16. Grensman A, Acharya BD, Wändell $P$, et al. Effect of traditional yoga, mindfulness-based cognitive therapy, and cognitive behavioral therapy, on health related quality of life: A randomized controlled trial on patients on sick leave because of burnout. BMC Complement Altern Med. Published online 2018. doi:10.1186/s12906-018-2141-9

17. Goldhagen BE, Kingsolver K, Stinnett SS, Rosdahl JA. Stress and burnout in residents: Impact of mindfulness-based resilience training. Adv Med Educ Pract. Published online 2015. doi:10.2147/AMEP.S88580 
18. Wen L, Sweeney TE, Welton L, Trockel M, Katznelson L. Encouraging Mindfulness in Medical House Staff via Smartphone App: A Pilot Study. Acad Psychiatry. Published online 2017. doi:10.1007/s40596-017-0768-3

19. MacCoon DG, Imel ZE, Rosenkranz MA, et al. The validation of an active control intervention for Mindfulness Based Stress Reduction (MBSR). Behav Res Ther. Published online 2012. doi:10.1016/j.brat.2011.10.011

20. Rosenkranz MA, Dunne JD, Davidson RJ. The next generation of mindfulness-based intervention research: what have we learned and where are we headed? Curr Opin Psychol. Published online 2019. doi:10.1016/j.copsyc.2018.12.022

21. Santorelli SF, Kabat-Zinn J. Mindfulness-based stress reduction (MBSR): Standards of practice. Mindfulness-Based Stress Reduct Stand Pract. Published online 2014.

22. Lim WY, Ong J, Ong S, et al. The Abbreviated Maslach Burnout Inventory Can Overestimate Burnout: A Study of Anesthesiology Residents. J Clin Med. Published online 2019. doi:10.3390/jcm9010061

23. www.mindgarden.com. https://www.mindgarden.com/315-mbi-human-services-survey-medicalpersonnel

24. Kalliokoski O, Jellestad FK, Murison R. A systematic review of studies utilizing hair glucocorticoids as a measure of stress suggests the marker is more appropriate for quantifying short-term stressors. Sci Rep. Published online 2019. doi:10.1038/s41598-019-48517-2

25. Meyer J, Novak M, Hamel A, Rosenberg K. Extraction and analysis of cortisol from human and monkey hair. J Vis Exp. Published online 2014. doi:10.3791/50882

\section{Tables}

Table 1. Study Timeline

\begin{tabular}{|c|c|c|c|c|c|c|}
\hline \multirow[b]{2}{*}{ Study Day } & \multicolumn{2}{|c|}{ Pre-Intervention } & \multicolumn{3}{|c|}{ Intervention Phase } & \multirow{2}{*}{$\begin{array}{l}\text { Post-Intervention } \\
720\end{array}$} \\
\hline & 1 & 90 & 180 & 360 & 540 & \\
\hline MBI-HSS & 口 & - & $\square$ & $\square$ & $\square$ & $\mathbf{\square}$ \\
\hline Hair Cortisol & 口 & 口 & $\square$ & $\square$ & $\mathbf{\square}$ & $\square$ \\
\hline
\end{tabular}

\section{Table 2. MBI-HSS Between \& Within Group Comparisons}




\begin{tabular}{|c|c|c|c|c|c|c|c|c|}
\hline & & Comparison & Ref. & Days & Difference & $\begin{array}{l}\text { Standard } \\
\text { Error }\end{array}$ & $\begin{array}{l}\mathrm{P}- \\
\text { value }\end{array}$ & $\begin{array}{l}\text { P-value for } \\
\text { interaction* }\end{array}$ \\
\hline \multirow[t]{3}{*}{ Group } & EETS & & & & 2.64 & 2.77 & 0.347 & \multirow{3}{*}{$\begin{array}{l}\text { All are not } \\
\text { statistically } \\
\text { significant }\end{array}$} \\
\hline & DTS & & & & -3.90 & 1.82 & 0.040 & \\
\hline & PATS & & & & 0.26 & 1.66 & 0.879 & \\
\hline Campaign & EETS & CA2 & CA1 & 0 & -0.19 & 2.98 & 0.950 & 0.048 \\
\hline \multirow{8}{*}{$\begin{array}{l}\text { by CA } \\
\text { year }\end{array}$} & & & & 180 & 7.81 & 3.73 & 0.047 & \\
\hline & & & & 360 & 8.08 & 4.75 & 0.101 & \\
\hline & DTS & $\mathrm{CA} 2$ & CA1 & 0 & -2.01 & 1.90 & 0.301 & 0.002 \\
\hline & & & & 180 & 5.40 & 2.25 & 0.024 & \\
\hline & & & & 360 & 0.84 & 2.74 & 0.762 & \\
\hline & PATS & CA2 & CA1 & 0 & -0.88 & 1.78 & 0.626 & 0.033 \\
\hline & & & & 180 & -5.85 & 2.23 & 0.015 & \\
\hline & & & & 360 & -6.35 & 2.84 & 0.034 & \\
\hline
\end{tabular}

Figures 


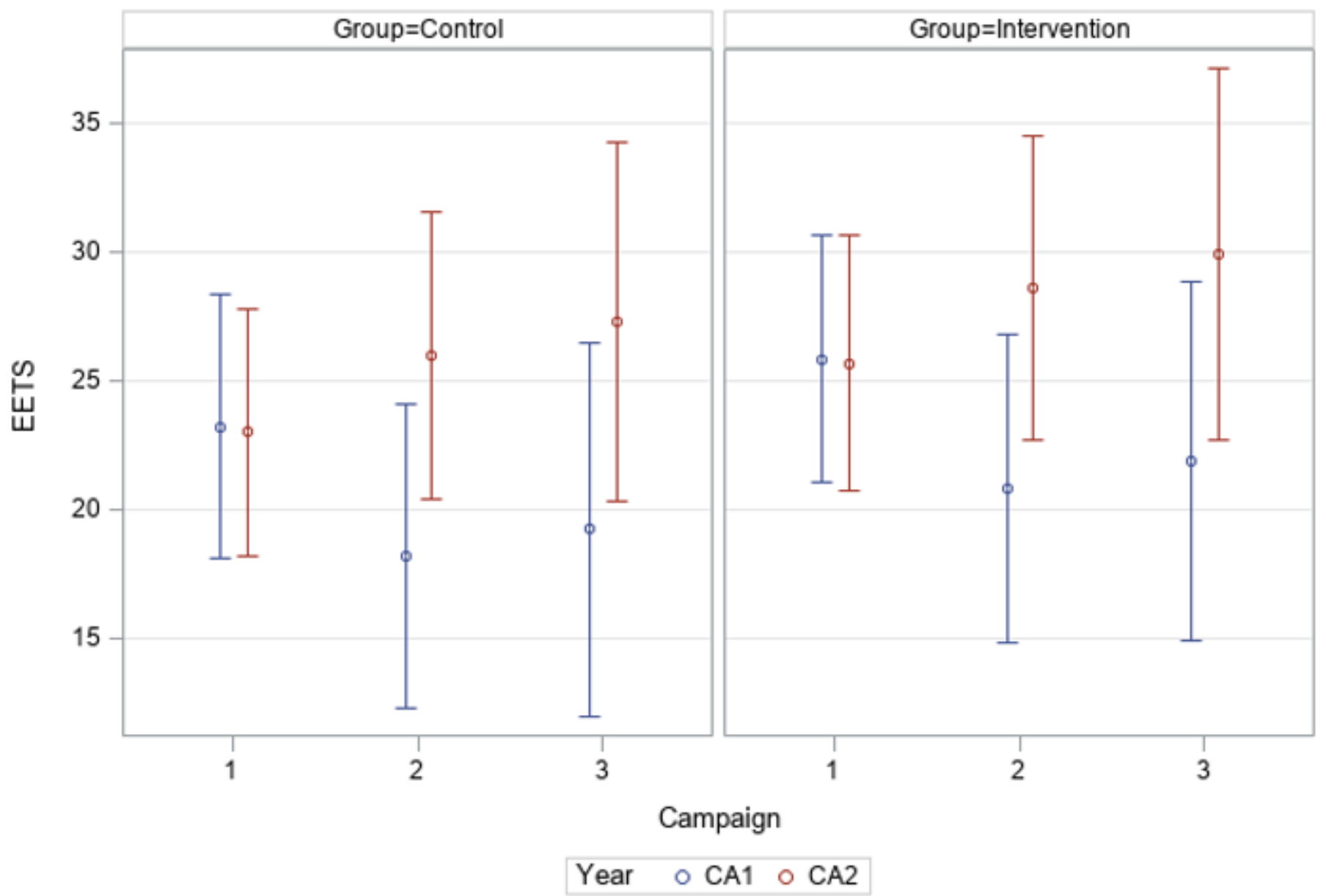

\section{Figure 1}

Emotional Exhaustion Total Score (EETS) Results. Emotional Exhaustion Total Scores (with $95 \% \mathrm{Cl}$ ) by Clinical Anesthesia Year over Campaign Time. Campaign $1=$ day 0 , Campaign $2=$ day 180, Campaign $3=$ day 360 . 


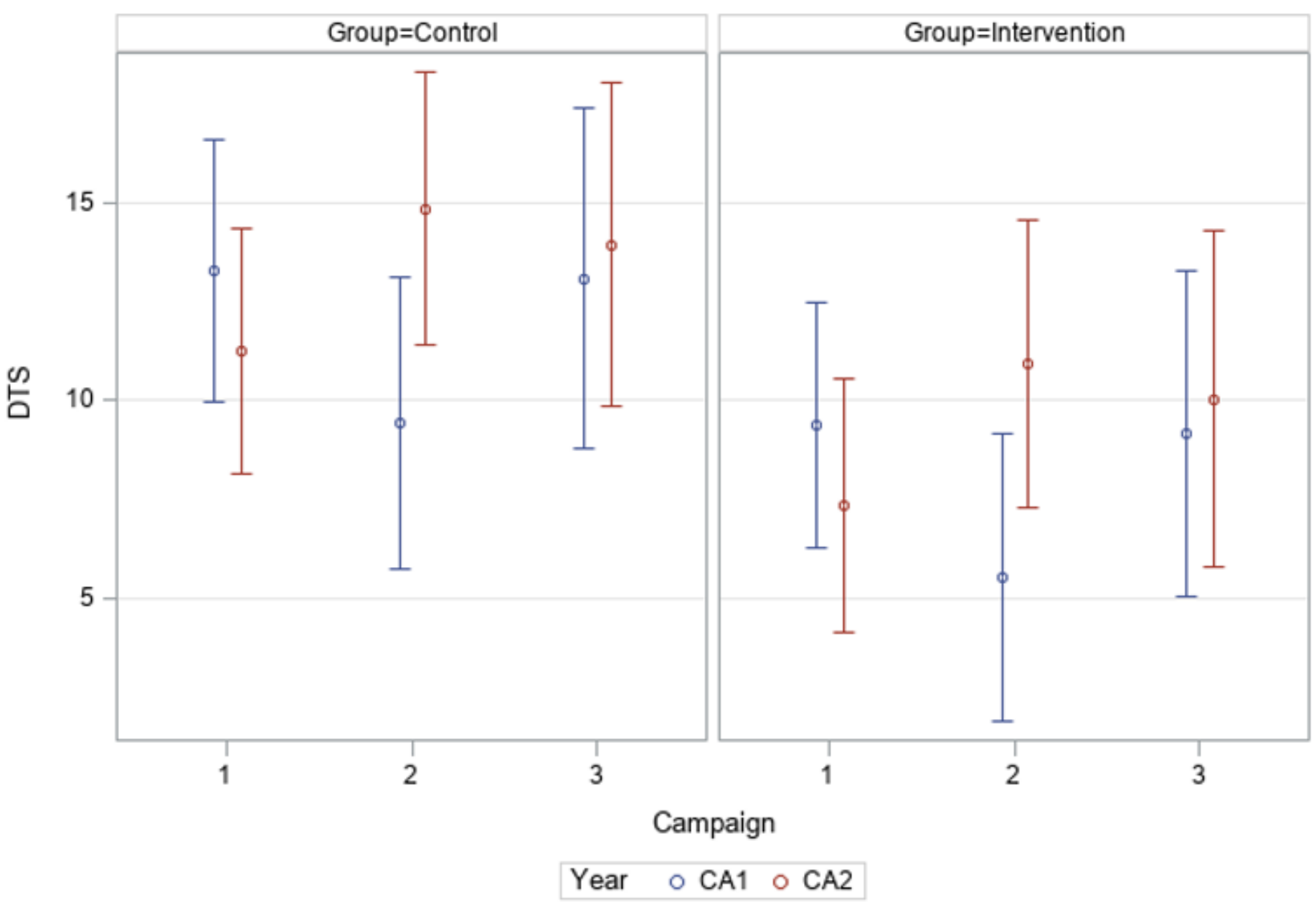

Figure 2

Depersonalization Total Score (DTS) Results. Depersonalization Total Scores (with 95\% Cl) by Clinical Anesthesia Year over Campaign Time. Campaign $1=$ day 0 , Campaign $2=$ day 180 , Campaign $3=$ day 360. 


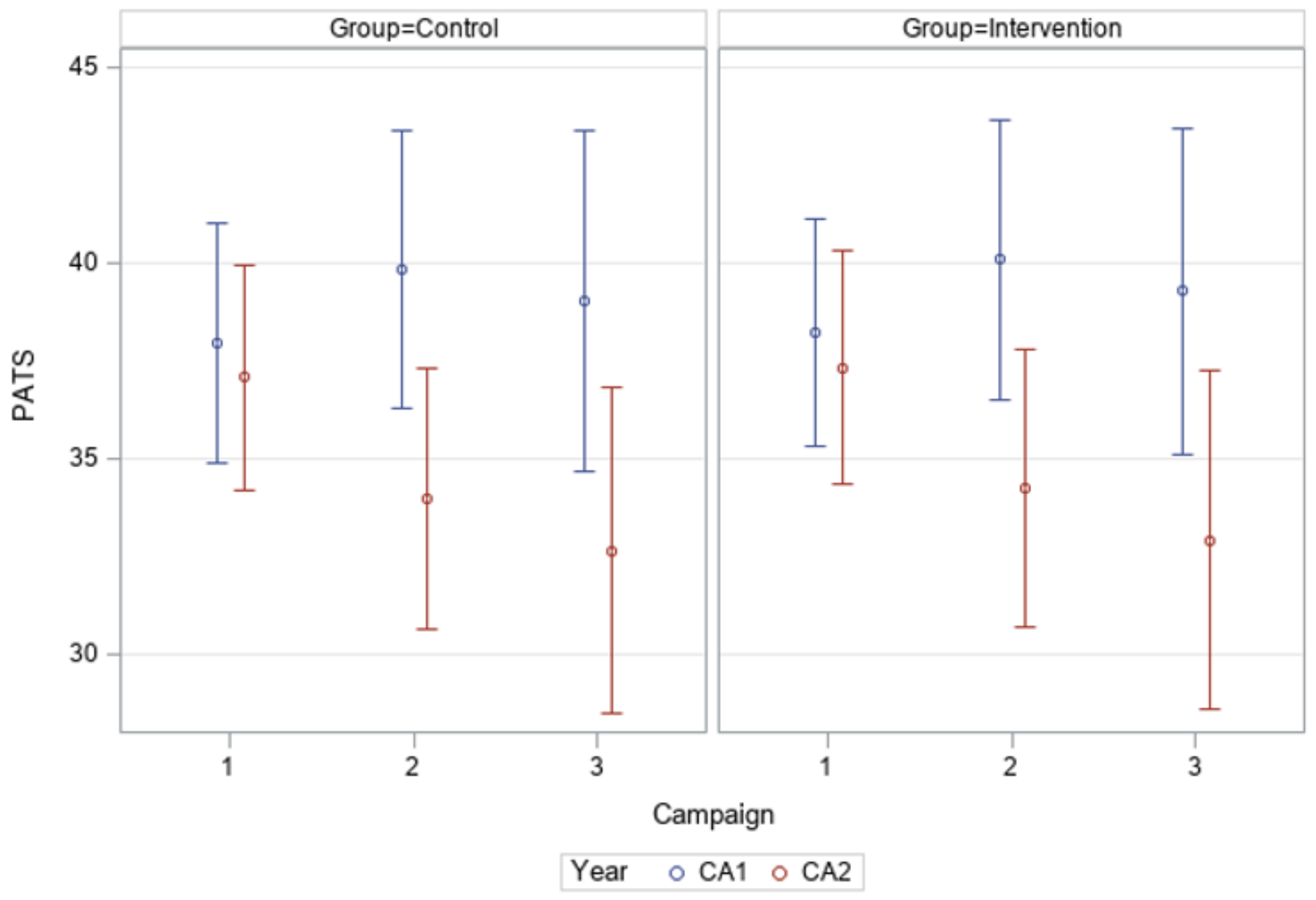

Figure 3

Personal Accomplishment Total Score (PATS) Results. Personal Accomplishment Total Scores (with 95\% $\mathrm{Cl}$ ) by Clinical Anesthesia Year over Campaign Time. Campaign $1=$ day 0 , Campaign $2=$ day 180 , Campaign $3=$ day 360 . 